Received: 2015.04.07

Accepted: 2015.04.22

Published: 2015.05.16

\title{
Effects of Ivabradine on Cardiac Electrophysiology in Dogs with Age-Related Atrial Fibrillation
}

Authors' Contribution: Study Design A Data Collection B Statistical Analysis C Data Interpretation D Manuscript Preparation E Literature Search F Funds Collection $G$
ABCDEFG 1 Yao-Dong Li*

ABCDEF 1 Yu-Tong Ji*

ABCDEFG 1 Xian-Hui Zhou

A 2 Tao Jiang

ABCDEF 1 Yi-fan Hong

C 1 Jin-Xin Li

F 1 Qiang Xing

F 1 Jian Xiong

B 1 Yueerguli Yusufuaji

A 1 Bao-Peng Tang
1 Department of Pacing and Electrophysiology, The First Affiliated Hospital of Xinjiang Medical University, Urumqi, Xinjiang, P.R. China

2 Clinical Research Institute, The First Affiliated Hospital of Xinjiang Medical University, Urumqi, Xinjiang, P.R. China
Corresponding Author: Source of support:

* Yao-dong Li and Yu-tong Ji is contributed equally to this work Bao-Peng Tang, e-mail: tangbaopeng@hotmail.com This study was supported by the National Natural Science Foundation of China (no. 81260069)

Background:

Material/Methods:

Results:

Conclusions:

MeSH Keywords:

Full-text PDF:
Ivabradine is an inhibitor of mixed $\mathrm{Na}^{+}-\mathrm{K}^{+}$current that could combine with $\mathrm{HCN}$ channels to reduce the transmembrane velocity of funny current (If), heart rate, and cardiac efficiency, and thus be used for the treatment of cardiovascular diseases such as chronic heart failure. As an ion channel blocker, Ivabradine is also a potential antiarrhythmic agent.

Twelve aging dogs (8-10 years old) underwent rapid atrial pacing for 2 months to induce age-related AF in this study. The dogs were randomly divided into the Ivabradine group and aging-AF group. The effects of Ivabradine on the electrophysiological parameters, including the effective refractory period (ERP) of the pulmonary veins and atrium, duration of $A F$, and inducing rate of $A F$, were investigated.

As compared to the aging-AF group, the ERPs of the left superior pulmonary vein $(139.00 \pm 4.18 \mathrm{~ms} v \mathrm{~s}$. $129.00 \pm 4.08 \mathrm{~ms}, \mathrm{P}=0.005)$ and left auricle $(135.00 \pm 3.53 \mathrm{~ms}$ vs. $122.00 \pm 4.47 \mathrm{~ms}, \mathrm{P}=0.001)$ were significantly increased, while the duration of $A F(46.60 \pm 5.07$ s vs. $205.40 \pm 1.14 \mathrm{~s}, \mathrm{P}=0.001)$ and inducing rate of $\mathrm{AF}(25 \%$ vs. $60 \%, P=0.001)$ were significantly decreased.

Ivabradine could effectively reduce the inducing rate of AF, and thus be used as an upstream drug for the prevention of age-related AF.

Aging • Arrhythmias, Cardiac • Atrial Fibrillation • Ivabradine

http://www.medscimonit.com/abstract/index/idArt/894320

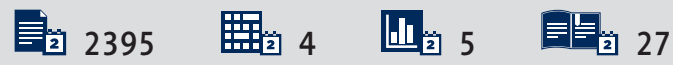




\section{Background}

Atrial fibrillation (AF) is one of the most common arrhythmias observed in clinical practice; it can lead to congestive heart failure, thromboembolism, and stroke. The incidence and mortality rates of AF are very high [1,2]. Epidemiological studies have shown that the incidence rate of AF doubles every 10 years. It is estimated that $84 \%$ of the patients with $\mathrm{AF}$ are over 65 years of age, and the incidence rate of AF is as high as $10 \%$ in people over 85 years of age $[3,4]$. Therefore, AF is considered as a major public health concern and risk factor in the aging population [5].

Funny current (If) is a mixed $\mathrm{Na}^{+}-\mathrm{K}^{+}$inward current mainly expressed in the sinoatrial node, and is a dominant factor for spontaneous depolarization of cardiac muscle in the diastolic phase [6]. Hyperpolarization-activated cyclic nucleotide-gated channels (HCN) are the molecular basis of If. The HCN family includes 4 members, among which HCN2 and HCN4 are highly expressed in cardiac tissues [7]. Previous studies have suggested that HCN channels mainly exist in the sinoatrial node and have low expression in normal myocardial tissue. However, recent studies have demonstrated that If also exists in other cardiac tissues, including atrium and ventricle, besides the sinoatrial node, which could trigger ectopic tachyarrhythmia [8].

It is well known that the incidence of sinoatrial node dysfunction (SND) increases with age and is accompanied by AF and other tachyarrhythmia [9]. Decreased functioning of the sinoatrial node facilitates electrical remodeling in the atrium and pulmonary veins, which could increase the local automaticity and triggered activity, and thus increase the risk of $\operatorname{AF}[10,11]$. Aging, as an independent risk factor, also can decrease the expression of If and HCN channels in the sinoatrial node and increase their expression in the atrium and pulmonary veins [12]. Our preliminary study showed that compared with the adult sinus dogs, the mRNA and protein levels of HCN2 and HCN4 in the sinoatrial node decreased in adult dogs with AF. In addition, the lower expression of HCN channels was more pronounced in aging dogs with AF [13], suggesting that HCN channels and If mediated by HCN channels could be potential mechanisms of age-related AF.

Ivabradine is a selective and specific If inhibitor that can affect the spontaneous depolarization of the cells in the fourth phase, and thus reduce cellular automaticity [14]. As an ion channel inhibitor, Ivabradine has potential antiarrhythmic effects. We hypothesized that Ivabradine could affect the electrophysiological features in dogs with age-related AF. In the present study, age-related AF was induced by rapid atrial pacing followed by the administration of Ivabradine to validate the hypothesis.

\section{Material and Methods}

\section{Animals}

Twelve healthy aging Beagle dogs, regardless of sex, were used in the present study. The mean body weight of the dogs was $13.40 \pm 1.14 \mathrm{~kg}$, and the mean age was $8.66 \pm 1.63$ years (the age of each dog was estimated by veterinarians according to the age evaluation criteria, including the status of the teeth, fur, muscle, and bone conformation). All dogs were housed and cared for in the Animal Experimental Center at the First Affiliated Hospital of Xinjiang Medical University. Echocardiography was used to exclude organic heart disease, and body surface electrocardiogram was used to confirm the sinus rate. All procedures were performed according to the Guide for the Care and Use of Laboratory Animals issued by the National Research Institution. The study design and processes were approved by the Animal Ethics Committee of the First Affiliated Hospital of Xinjiang Medical University (IACUC), granting ethics approval for use of up to 15 dogs.

\section{Surgery of age-related AF}

Basal anesthesia was induced by injecting $30 \mathrm{mg} / \mathrm{kg}$ of sodium pentobarbital followed by implantation of a pacemaker through the external jugular vein according to previously described methods [15]. All dogs underwent rapid right atrial pacing at a frequency of 600 beats/min for 2 months to induce the age-related AF model. The dogs were randomly divided into 2 groups - the Ivabradine group and the aging-AF group (according to whether the Ivabradine intervention to divide group) - with 6 animals in each group. Ivabradine capsule $(4 \mathrm{mg} / \mathrm{kg} / \mathrm{d}$ ) was orally administered to the dogs in the Ivabradine group during the pacing for 2 months [16,17], while no drug was provided to the aging-AF group.

\section{Data collection for cardiac electrophysiology}

Inducing rate and duration of AF: S1S1 program was used for the stimulation before and at 2 months after the pacing, respectively. The voltage used was twice the diastolic threshold. Each stimulation was for $30 \mathrm{~s}$ followed by an interval of 2 min. A total of 10 stimulations were continuously performed on each dog, and the data on AF were recorded. Multi-lead electrophysiological instrument showing chaotic atrial wave with no P-wave, and f-waves of different sizes, intervals, and shapes that lasted for at least $10 \mathrm{~s}$ was considered as successful induction of AF. The duration of AF was the time from the successful induction to the reversal of $f$-wave to sinus P-wave.

Effective refractory period (ERP) of pulmonary veins and atrium: Pre-program stimulation (S1S2) was used for the pulmonary veins, as well as the left and right auricles at two months 


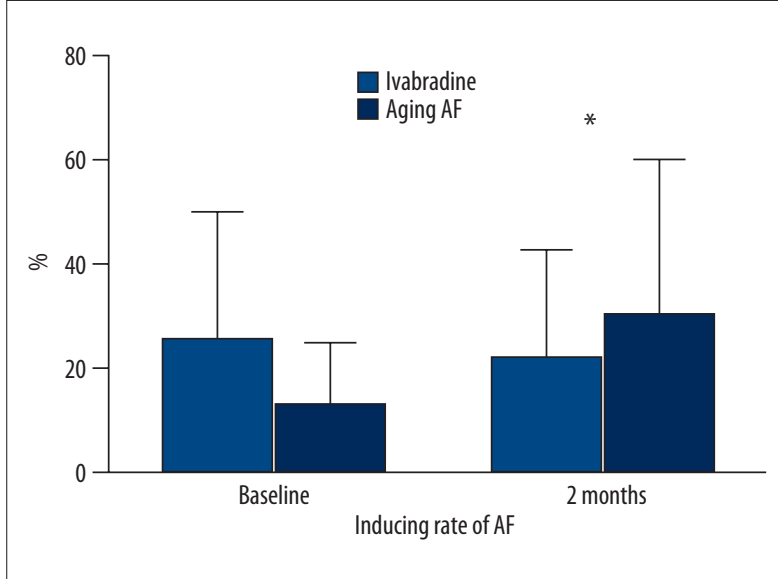

Figure 1. Inducing rate of AF in the Ivabradine and aging-AF groups. A significant difference in the inducing rate of AF was found at baseline and 2 months after pacing. ${ }^{*} P<0.05$.

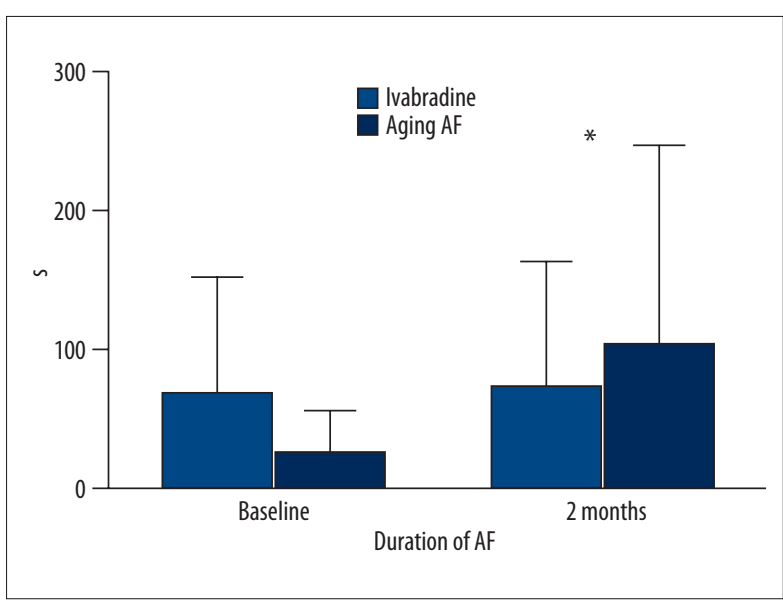

Figure 2. Duration of AF in the Ivabradine and aging-AF groups. A significant difference in the duration of $A F$ was found at baseline and 2 months after pacing. ${ }^{*} P<0.05$.

Table 1. Inducing rate and duration of AF.

\begin{tabular}{|ccccc} 
& \multicolumn{2}{c}{ Inducing rate of AF (\%) } & \multicolumn{2}{c|}{ Duration of AF (s) } \\
\hline & Baseline & $\mathbf{2}$ months & Baseline & $\mathbf{2}$ months \\
\hline Ivabradine group & 50 & 25 & $127.40 \pm 9.63$ & $46.60 \pm 5.07$ \\
\hline Aging-AF group & 43 & 60 & $137.60 \pm 9.39$ & $205.40 \pm 3.14$ \\
\hline$P$ & 0.929 & 0.001 & 0.129 & 0.001 \\
\hline
\end{tabular}

after the pacing to measure the ERP. The stimulus threshold was twice the diastolic threshold. One premature stimulus (S2) was applied per eight basal stimulations of S1S1. The scanning was performed with the initial pacing-length of $250 \mathrm{~ms}$ and shortened by $5 \mathrm{~ms}$ until capture was lost, and the longest S1S2 interval was recorded as the ERP. The ERPs of the pulmonary veins and auricles were measured with the basal stimulus of $400,350,300$, and $250 \mathrm{~ms}$, respectively, with three measurements for each site per dog, and the mean values were calculated for the analyses. 1) dispersion of ERP was recorded as the difference between the maximum and minimum values of ERP of each site; 2) ERP shortened with the decrease of the base perimeter was considered as ERP frequency adaptation, otherwise it was considered as rate in adaptation of ERP. (ERP 350-ERP300)/50 ms was used to determine the rate adaptation of ERP [18].

\section{Statistical analyses}

The data were expressed as mean \pm standard deviation (SD). Chi-square test was used to compare the inducing rate of AF. Analysis of variance was used to compare the ERPs of the pulmonary veins and atrium, and the duration of AF. The statistical analyses were performed using SPSS 17.0 software. $P<0.05$ was considered to be statistically significant.

\section{Results}

\section{Effects of Ivabradine on the inducing rate and duration of AF}

All dogs were stimulated with a frequency of 600 beats $/ \mathrm{min}$ for 2 months. The inducing rate of AF decreased by $25 \%$ in the Ivabradine group but increased by $17 \%$ in aging-AF group. The inducing rate of AF was $50 \%$ and $43 \%$ in the Ivabradine and aging-AF groups at the baseline $(P=0.292)$, and $25 \%$ and $60 \%$ at two months after the pacing $(P=0.001)$, respectively. During the two months of pacing, the duration of AF decreased from $127.40 \pm 9.63 \mathrm{~s}$ to $46.60 \pm 5.07 \mathrm{~s}$ in the Ivabradine group, but increased from $137.60 \pm 9.39 \mathrm{~s}$ to $205.40 \pm 1.14 \mathrm{~s}$ in agingAF group. In addition, the longest duration of $A F$ in aging-AF group was about 1000s. The difference in the duration of AF was insignificant between the two groups before the pacing

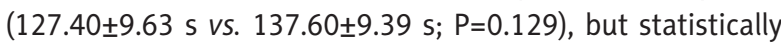
significant at two months after the pacing $(46.60 \pm 5.07 \mathrm{~s} v \mathrm{~s}$. $205.40 \pm 1.14 \mathrm{~s} ; \mathrm{P}=0.001)$. These findings suggest that Ivabradine reduces the inducing rate as well as the duration of $A F$ after the induction (Figures 1, 2; Table 1). 


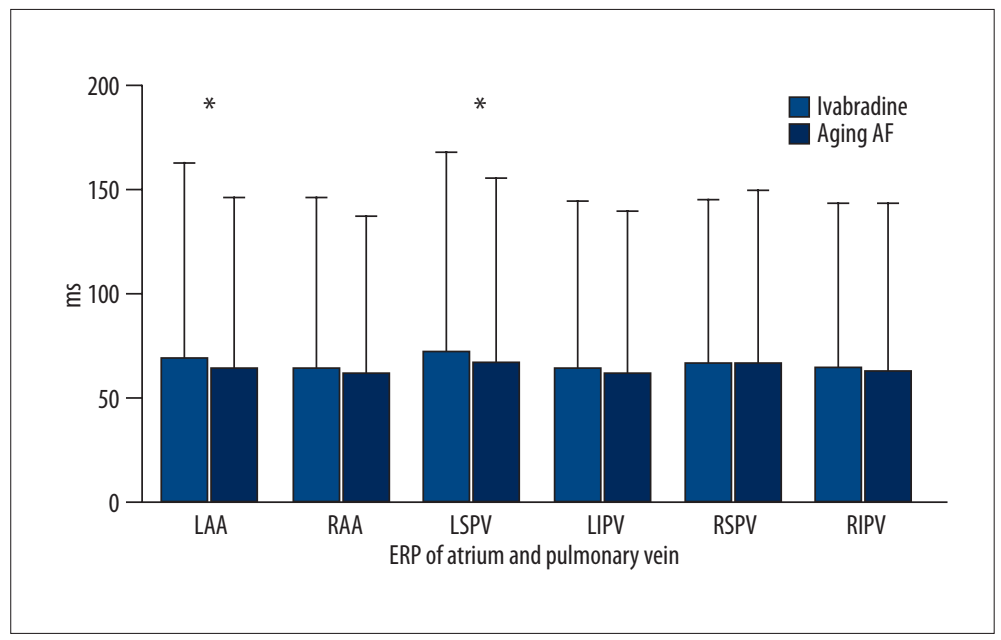

Figure 3. ERP in atrium and pulmonary vein. A significant difference in the ERP of the auricle and pulmonary veins was found between the 2 groups. * $P<0.05$. LAA - left auricle; RAA - right auricle; LSPV - left superior pulmonary vein; LIPV- left inferior pulmonary vein; RSPV - right superior pulmonary vein; RIPV - right inferior pulmonary vein; ERP - effective refractory period

Table 2. ERP of atrium and pulmonary vein.

\begin{tabular}{|c|c|c|c|c|c|c|}
\hline & LAA (ms) & RAA (ms) & LSPV (ms) & LIPV (ms) & RSPV (ms) & RIPV (ms) \\
\hline Ivabradine group & $135.00 \pm 3.53$ & $121.00 \pm 4.18$ & $139.00 \pm 4.18$ & $120.00 \pm 5.00$ & $121.00 \pm 11.40$ & $120.00 \pm 7.07$ \\
\hline Aging-AF group & $122.00 \pm 4.47$ & $115.00 \pm 7.90$ & $129.00 \pm 4.08$ & $116.00 \pm 6.51$ & $125.00 \pm 7.90$ & $119.00 \pm 4.18$ \\
\hline$P$ & 0.001 & 0.172 & 0.005 & 0.308 & 0.537 & 0.792 \\
\hline
\end{tabular}

LAA - left auricle; RAA - right auricle; LSPV - left superior pulmonary vein; LIPV - left inferior pulmonary vein; RSPV - right superior pulmonary vein; RIPV - right inferior pulmonary vein; ERP - effective refractory period.

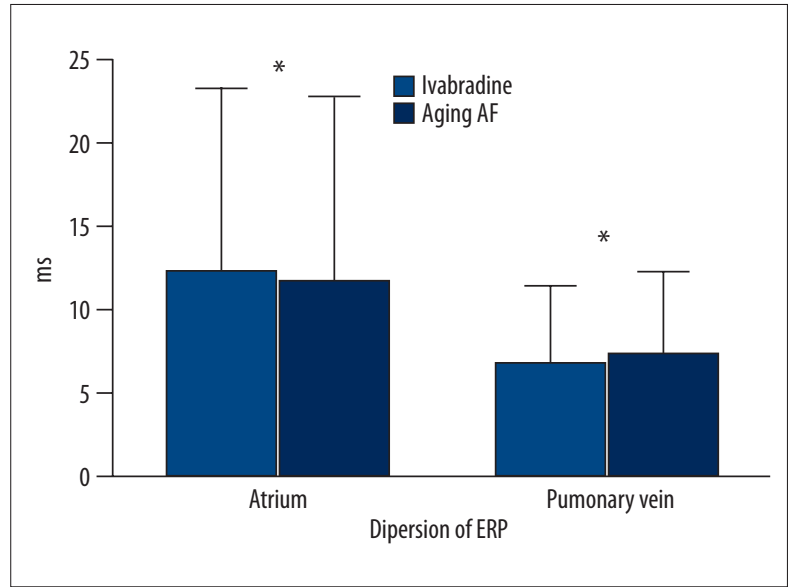

Figure 4. Variations of the ERPs in Atrium and Pulmonary vein. A significant difference in the ERPs of the left auricle and left superior pulmonary vein was seen between the 2 groups. ${ }^{*} P<0.05$.

\section{Effects of Ivabradine on the ERP of the pulmonary veins and atrium}

After basal stimulation with the same frequencies, the ERP decreased gradually with the decrease in basal stimulus at each site in both groups. After the administration of Ivabradine, the ERP of the left auricle $(135.00 \pm 3.53 \mathrm{~ms}$ vs. $122.00 \pm 4.47 \mathrm{~ms}$;
Table 3. Dispersion of ERP in atrium and pulmonary vein.

\begin{tabular}{|ccc} 
& Atrium (ms) & Pulmonary vein (ms) \\
\hline Ivabradine group & $20.00 \pm 4.47$ & $19.16 \pm 3.76$ \\
\hline Aging-AF group & $10.00 \pm 3.16$ & $10.83 \pm 3.76$ \\
\hline$P$ & 0.001 & 0.003 \\
\hline
\end{tabular}

$\mathrm{P}=0.001)$ and left superior pulmonary vein $(139.00 \pm 4.18 \mathrm{~ms}$ vs. $129.00 \pm 4.08 \mathrm{~ms} ; \mathrm{P}=0.005)$ increased significantly. However, no significant difference was found in the ERPs of the right auricle $(121.00 \pm 4.18 \mathrm{~ms}$ vs. $115.00 \pm 7.90 \mathrm{~ms} ; \mathrm{P}=0.172)$, right superior pulmonary vein $(121.00 \pm 11.40 \mathrm{~ms}$ vs. $125.00 \pm 7.90 \mathrm{~ms}$; $\mathrm{P}=0.537)$, right inferior pulmonary vein $(120.00 \pm 7.07 \mathrm{~ms}$ vs. $119.00 \pm 4.18 \mathrm{~ms} ; \mathrm{P}=0.792)$, and left inferior pulmonary vein $(120.00 \pm 5.00$ ms vs. $116.00 \pm 6.51 \mathrm{~ms} ; \mathrm{P}=0.308)$ between the two groups (Figure 3, Table 2). The variation of ERP was $(20.00 \pm 4.47 \mathrm{~ms}$ vs. $10.00 \pm 3.16 \mathrm{~ms} ; \mathrm{P}=0.001)$ in the atrium and $(19.16 \pm 3.76 \mathrm{~ms}$ vs. $10.83 \pm 3.76 \mathrm{~ms} ; \mathrm{P}=0.003)$ in the pulmonary veins, respectively (Figure 4 , Table 3 ). As compared to aging-AF group, the ERP frequency adaptation increased in the Ivabradine group, and the difference in the left auricle $(P=0.05)$ and left superior pulmonary vein $(P=0.046)$ was statistically significant between the two groups (Figure 5, Table 4). 


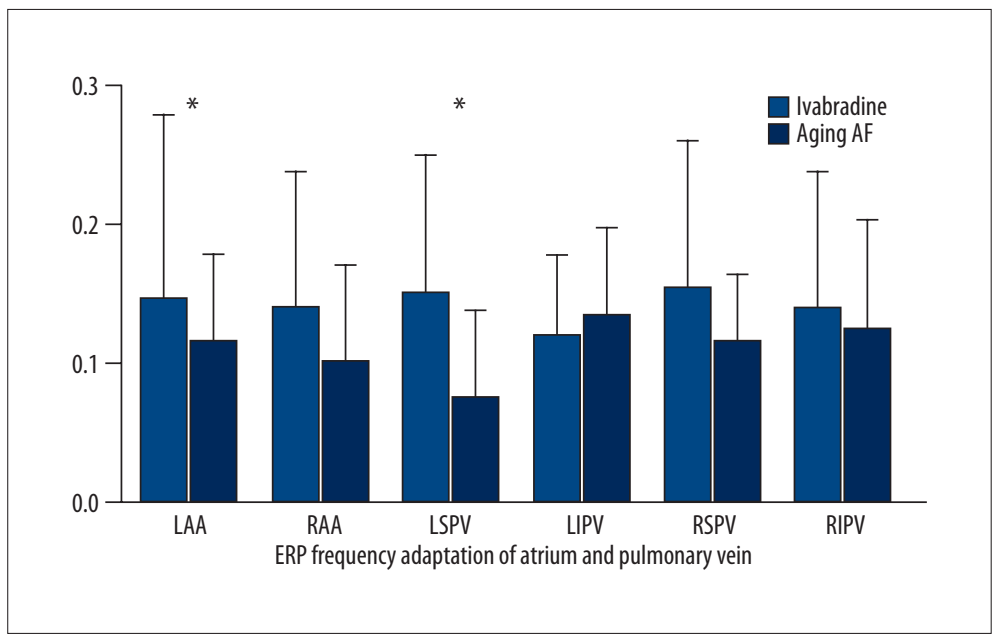

Figure 5. ERP frequency adaptations in Atrium and Pulmonary vein. The ERP frequency adaptations in the auricles and pulmonary veins were significantly different between the 2 groups.

${ }^{*} P<0.05$. LAA - left auricle; RAA - right auricle; LSPV - left superior pulmonary vein; LIPV - left inferior pulmonary vein; RSPV - right superior pulmonary vein; RIPV - right inferior pulmonary vein; ERP - effective refractory period.

Table 4. ERP frequency adaptation in atrium and pulmonary vein.

\begin{tabular}{lcccccc} 
& LAA & RAA & LSPV & LIPV & RSPV & RIPV \\
\hline Ivabradine group & $0.24 \pm 0.05$ & $0.21 \pm 0.07$ & $0.22 \pm 0.08$ & $0.16 \pm 0.08$ & $0.23 \pm 0.08$ & $0.21 \pm 0.07$ \\
\hline Aging-AF group & $0.16 \pm 0.07$ & $0.15 \pm 0.05$ & $0.12 \pm 0.03$ & $0.18 \pm 0.09$ & $0.15 \pm 0.08$ & $0.18 \pm 0.07$ \\
\hdashline$P$ & 0.05 & 0.11 & 0.046 & 0.756 & 0.111 & 0.461 \\
\hline
\end{tabular}

LAA - left auricle; RAA - right auricle; LSPV - left superior pulmonary vein; LIPV - left inferior pulmonary vein; RSPV - right superior pulmonary vein; RIPV - right inferior pulmonary vein; ERP - effective refractory period.

\section{Discussion}

The present study showed that Ivabradine not only increased the ERPs of the left pulmonary vein and left atrium, but also reduced the duration and inducing rate of $A F$ after induction in a dog model of age-related AF.

\section{Role of If in age-related AF}

Several studies have shown that If and HCN channels are closely related to tachyarrhythmia. In some pathological conditions, the abnormal expressions or structures of the HCN channels in the cardiac muscles could lead to increased or decreased If, and finally induce arrhythmia. Chen et al. [19] have shown that pulmonary vein cardiomyocytes are rich in If, which increased after rapid atrial pacing. Our previous studies have also shown that If was closely related to AF, and the mRNA and protein levels of $\mathrm{HCN}$ channels increased significantly in the atrial muscles of patients with AF [20]. The decrease in the sinoatrial node function, increase in the activities of ectopic pacemakers including atrium and pulmonary veins, and increase of local automaticity with age could increase the risk of ectopic arrhythmia [21]. Li et al. [22] showed that the density of If was significantly higher in the cardiac myocytes from pulmonary vein sleeves of aged dogs with AF than in the ones with sinus rate. Our previous studies have shown that aging could alter the electrophysiological parameters of the atrium in dogs, decrease the sinoatrial node functions, and induce reversed expression of mRNAs and proteins of HCN2 and HCN4 in the sinoatrial node, pulmonary veins, and atrium. The alterations in the electrophysiological characteristics and HCN channels in the atrium could be associated with the development and continuance of age-related AF [13].

\section{Effects of Ivabradine on age-related AF}

Ivabradine is an inhibitor of If in the sinoatrial node, and can reduce heart rate and cardiac efficiency. It is mainly used for the treatment of several cardiovascular diseases, including chronic heart failure and stable angina pectoris, in clinical practice $[23,24]$. As an ion channel inhibitor, Ivabradine could also have potential antiarrhythmic effects. In a study by Suenari et al. [25], pulmonary vein sleeve cells from rabbits were dissociated for patch clamp examinations, and the existence of spontaneous action potential mediated by If was found, which decreased after the administration of Ivabradine. In another study, a model of chronic AF was induced by rapid atrial pacing in aged dogs, which showed that Ivabradine could inhibit the frequency of spontaneous action potential in the pulmonary vein sleeve cells [22]. Specimens from the right auricle were obtained from patients requiring cardiac surgeries, and Ivabradine was added during patch clamp examinations in a 
study by Chemaly et al. [26], which showed that Ivabradine could inhibit the If in the non-pacemaker cells in the atrium similar to the effects in the pacemaker cells in the sinoatrial node, suggesting that Ivabradine could potentially affect the atrial electrical activities. These findings suggest that Ivabradine could reduce the cellular automaticity induced by If by inhibiting the HCN channels and the mediated If in pulmonary veins and atrium, and thus potentially prevent the development of AF.

In the present study, dogs with age-related AF induced by rapid atrial pacing, were administered Ivabradine for intervention. The results showed that Ivabradine could increase the ERPs in the left atrium and left superior pulmonary vein, reduce the duration of AF after induction, and significantly decrease the inducing rate of $\mathrm{AF}$ (the incidence rate of $\mathrm{AF}$ decreased from $50 \%$ to $25 \%$ ). However, the underlying mechanisms remain unclear. We speculated that Ivabradine could inhibit the If in the atrium and pulmonary veins and thus exert its preventive effects, since the development of age-related AF is related to the triggered activity induced by the increase of If in pulmonary veins. Ivabradine could inhibit the transmembrane velocity of If in the pulmonary veins, reduce the frequency of spontaneous action potential, and thus decrease the risk of age-related AF. Therefore, Ivabradine has potential effects in preventing age-related AF.

However, a meta-analysis performed by Martin et al. [27] showed that Ivabradine could increase the risk of incident AF, and patients receiving Ivabradine had $15 \%$ higher risk of developing $A F$, which was the highest among previous studies, as compared to the control group. Ivabradine was also found to decrease the sinoatrial functions while inhibiting the pacing current, and thus increase the risk of AF, which was contrary to our findings. The present study showed that Ivabradine

\section{Reference:}

1. Chimenti C, Russo M A, Carpi A et al: Histological substrate of human atrial fibrillation. Biomed Pharmacother, 2010; 64(3): 177-83

2. Piccini JP, Hammill BG, Sinner MF et al: Incidence and prevalence of atrial fibrillation and associated mortality among Medicare beneficiaries, 19932007. Circ Cardiovasc Qual Outcomes, 2012; 5(1): 85-93

3. Lee GA, Stub D, Ling H: Atrial fibrillation in the elderly - not a benign condition. Int Emerg Nurs, 2012; 20(4): 221-27

4. Naderi S, Wang Y, Miller AL et al: The impact of age on the epidemiology of atrial fibrillation hospitalizations. Am J Med, 2014; 127(2): 158.e1-7

5. Lip GY, Tse HF: Management of atrial fibrillation. Lancet, 2007; 370: 604-18

6. Xue X, Yan GX: The funny current (If): is it a novel antiarrhythmic target to treat atrial fibrillation? J Cardiovasc Electrophysiol, 2012; 23(2): 207-8

7. Michels G, Er F, Khan I et al: Single-channel properties support a potential contribution of hyperpolarization-activated cyclic nucleotide-gated channels and If to cardiac arrhythmias. Circulation, 2005; 111: 399-404

8. Santoro B, Liu DT, Yao H et al: Identification of a gene encoding a hyperpolarization-activated pacemaker channel of brain. Cell, 1998; 93(5): 717-29

9. Semelka M, Gera J, Usman S: Sick sinus syndrome: a review. Am Fam Physician, 2013; 87(10): 691-96 could decrease the incidence of age-related AF, which could be due to inhibition of the If in the pulmonary veins. However, the present study was performed in a dog model of age-related AF induced by rapid atrial pacing; therefore, the effects of inhibiting the heart rate from the sinoatrial node and thus increasing the risk of AF might not be exerted.

\section{Limitations}

Only electrophysiological parameters were examined in the present study to evaluate the relationships between Ivabradine and $A F$, while the protein levels of single-cell ion channels and $\mathrm{HCN}$ channels were measured for the If. Therefore, this study provides some evidence but cannot be directly used in clinical practice. Additional studies are needed to investigate the effects of Ivabradine on the pattern changes of If, as well as the protein and mRNA levels of HCN channels, to understand its effects on age-related AF.

\section{Conclusions}

Ivabradine could significantly increase the ERPs of the left pulmonary veins and left atrium, shorten the duration of AF after the induction, and reduce the inducing rate of $A F$ in a dog model of age-related AF induced by rapid atrial pacing. Therefore, Ivabradine has potential protective effects against AF.

\section{Acknowledgments}

We thank the staff of the Animal Experimental Center at the First Affiliated Hospital of Xinjiang Medical University for their support and collaboration. We also thank the colleagues in the department and our laboratory for their help.

10. Li G, Liu E, Liu T et al: Atrial electrical remodeling in a canine model of sinus node Dysfunction. Int J Cardiol, 2011; 146(1): 32-36

11. Honjo $\mathrm{H}$, Boyett $\mathrm{MR}$, Niwa $\mathrm{R}$ et al: Pacing-induced spontaneous activity in myocardial sleeves of pulmonary veins after treatment with ryanodine. Circulation, 2003; 107(14): 1937-43

12. Yeh YH, Burstein B, Qi XY et al: Funny current downregulation and sinus node dysfunction associated with atrial tachyarrhythmia: a molecular basis for tachycardia-bradycardia syndrome. Circulation, 2009; 119: 1576-85

13. Li YD, Hong YF, Zhang Y et al: Association between reversal in the expression of hyperpolarization-activated cyclic nucleotide-gated (HCN) channel and age-related atrial fibrillation. Med Sci Monit, 2014; 20: 2292-97

14. Bucchi A, Tognati A, Milanesi R et al: Properties of ivabradine-induced block of HCN1 and HCN4 pacemaker channels. J Physiol, 2006; 572(2): 335-46

15. Li Y-d, Hong Y-f, Zhou X-h et al: Development of a living canine model of persistent atrial fibrillation by animal pacemaker implantation using four different methods. Experimental and Clinical Cardiology, 2014; 20(10) 5795-812

16. Kuwabara Y, Kuwahara K, Takano M et al: Increased expression of HCN channels in the ventricular myocardium contributes to enhanced arrhythmicity in mouse failing hearts. J Am Heart Assoc, 2013; 2(3): e000150 
17. Ping X: Animal management and operation of the technical specifications. China Shanghai Science and Technology Press, 2007

18. Kurita Y, Mitamura H, Shimshita-Takeshita A et al: Dally oral verapamil before but not after rapid atrial excitation prevents electrical remodeling Cardiovasc Res, 2002; 54: 447-55

19. Chen YJ, Chen SA, Chen YC et al: Effects of rapid atrial pacing on the arrhythmogenic activity of single cardiomyocytes from pulmonary veins: implication in initiation of atrial fibrillation. Circulation, 2001; 104(23): 2849-54

20. Zhang J, Gan T, Tang B: Studies in patients with valvular heart disease, left atrial fibrillation HCN channels and 5-HT4-R expression. Journal of Cardiac Arrhythmias, 2012; 16(1): 51-55

21. Ramdjan TT, van der Does LJ, Knops $P$ et al: Right versus left atrial pacing in patients with sick sinus syndrome and paroxysmal atrial fibrillation (Riverleft study): study protocol for randomized controlled trial. Trials, 2014 15: 445
22. Li JY, Wang HJ, Xu B et al: Hyperpolarization activated cation current I(f) in cardiac myocytes from pulmonary vein sleeves in the canine with atrial fibrillation. J Geriatr Cardiol, 2012; 9(4): 366-74

23. Camm AJ, Lau CP: Electrophysiological effects of a single intravenous administration of ivabradine (S 16257) in adult patients with normal baseline electrophysiology. Drugs R D, 2003; 4(2): 83-89

24. Urbanek I, Kaczmarek K, Cygankiewicz I et al: Risk-benefit assessment of ivabradine in the treatment of chronic heart failure. Drug Healthc Patient Saf, 2014; 6: 47-54

25. Suenari K, Cheng CC, Chen YC et al: Effects of Ivabradine on the pulmonary vein electrical activity and modulation of pacemaker currents and calcium homeostasis. J Cardiovasc Electrophysiol, 2012; 23(2): 200-6

26. El Chemaly A, Magaud $C$, Patri $S$ et al: The heart rate-lowering agent ivabradine inhibits the pacemaker current I(f) in human atrial myocytes. Cardiovasc Electrophysiol, 2007; 18(11): 1190-96

27. Martin RI, Pogoryelova O, Koref MS et al: Atrial fibrillation associated with ivabradine treatment: meta-analysis of randomized controlled trials. Heart, 2014; 100(19): 1506-10 